\title{
Preconception Alcohol Increases Offspring Vulnerability to Stress
}

\author{
Shaima Jabbar ${ }^{1,2}$, Lucy G Chastain', Omkaram Gangisetty', Miguel A Cabrera ${ }^{1,2}$, Kamil Sochacki' and \\ Dipak K Sarkar*,ı \\ 'The Endocrine Program, Department of Animal Sciences, Rutgers, The State University of New Jersey, New Brunswick, NJ, USA; ${ }^{2}$ Endocrinology \\ and Animal Biosciences Graduate Program, Rutgers, The State University of New Jersey, New Brunswick, NJ, USA
}

\begin{abstract}
The effect of preconception drinking by the mother on the life-long health outcomes of her children is not known, and therefore, in this study using an animal model, we determined the impact of preconception alcohol drinking of the mother on offspring stress response during adulthood. In our preconception alcohol exposure model, adult female rats were fed with $6.7 \%$ alcohol in their diet for 4 weeks, went without alcohol for 3 weeks and were bred to generate male and female offspring. Preconception alcohol-exposed offsprings' birth weight, body growth, stress response, anxiety-like behaviors, and changes in stress regulatory gene and protein hormone levels were evaluated. In addition, roles of epigenetic mechanisms in preconception alcohol effects were determined. Alcohol feeding three weeks prior to conception significantly affected pregnancy outcomes of female rats, with respect to delivery period and birth weight of offspring, without affecting maternal care behaviors. Preconception alcohol negatively affected offspring adult health, producing an increased stress hormone response to an immune challenge. In addition, preconception alcohol was associated with changes in expression and methylation profiles of stress regulatory genes in various brain areas. These changes in stress regulatory genes were normalized following treatment with a DNA methylation blocker during the postnatal period. These data highlight the novel possibility that preconception alcohol affects the inheritance of stress-related diseases possibly by epigenetic mechanisms.

Neuropsychopharmacology (2016) 4I, 2782-2793; doi: I0.1038/npp.2016.92; published online 6 July 2016
\end{abstract}

\section{INTRODUCTION}

The National Institute on Alcohol Abuse and Alcoholism estimated that $60 \%$ of U.S. women have at least one drink a year. Among those who drank, the prevalence of binge drinking was $15.0 \%$ among nonpregnant women and $1.4 \%$ among pregnant women. In the US, the prevalence of alcohol use disorders in women aged 18 years and older is about 4.6\% (SAMHSA, 2014). A recent perspective cohort study in Britain showed nearly two-thirds of women before pregnancy reported alcohol intakes of about 2 units ( $16 \mathrm{~g}$ alcohol) per week (Nykjaer et al, 2014). In a study determining the prevalence of lifetime and 7-day daily alcohol consumption in a community sample of Nigerians daily alcohol use has been reported to occur in 3.6\% women (Lasebikan and Gureje, 2015). Although the detrimental effects of prenatal alcohol on offspring health is known, the effects of preconception parental alcohol use on offspring is relatively unexplored. Heritability of alcohol-use disorders from paternal origin has been demonstrated (Finegersh et al, 2015). However, the effect of preconception drinking by the

* Correspondence: Dr DK Sarkar, Endocrinology and Animal Biosciences Graduate Program, Rutgers, The State University of New Jersey, 67 Poultry Farm Lane, New Brunswick, NJ, USA, Tel: 848932 I529, Fax: 732932 4134, E-mail: sarkar@aesop.rutgers.edu

Received 10 February 2016; revised 6 June 2016; accepted 8 June 20 I6; accepted article preview online 14 June 2016 mother on the life-long health outcome of her babies is not known, despite evidence for higher risk of having babies with lower birth weight and birth centile (Nykjaer et al, 2014) and increased risk for mental retardation (Roeleveld et al, 1992).

A rapidly accumulating body of evidence indicates that many diseases must be understood in a life-long perspective, as trajectories start at pre- or during conception and surface upon clinical detection decades later (Dover, 2009). Also, research into heritability of complex diseases has implicated transmission of epigenetic variants in the development of neuroendocrine phenotypes, including anxiety behaviors and stress responsivity (Malan-Müller et al, 2014). Furthermore, heritable deficiencies in specific gene function in gametes have been demonstrated (Dann et al, 2006).

In this study, we determined the impact of preconception alcohol drinking by the mother on pregnancy outcomes and on stress-related changes in offspring at multiple levels of analysis including endocrine, molecular, and behavioral levels using the rat as an animal model. We also investigated the epigenetic mechanisms underlying heritable stressrelated phenotypes.

\section{MATERIALS AND METHODS}

\section{Animal Feeding and Treatments}

All rat studies were performed with approved protocols in compliance with Association for the Assessment and 
Accreditation of Laboratory Animal Care and the Rutgers University. Fisher-344 strain rats were obtained from Charles River (Wilmington, MA), housed in controlled conditions with a 12-h light/dark cycle at a constant temperature $22^{\circ} \mathrm{C}$ throughout the study. Sixty adult female rats were assigned to one of three treatments for a period of 30 days: (1) the control group receiving rat chow and water ad libitum (AD; $n=18$; body weight, 128.6 \pm 3.1 ), (2) the alcohol fed group (AF; $n=24$; body weight, $130.6 \pm 2.7)$ receiving a liquid diet containing ethanol (6.7\%), or (3) the pair fed (PF; $n=18$; body weight, $133.3 \pm 3.3)$ group receiving an isocaloric liquid control diet (BioServ, Flemington, NJ) to match the daily intake of the rats receiving the alcohol-containing diet (Govorko et al, 2012). There was no significant body weight difference between these three groups in the beginning of the experiment $(F(2,57)=0.5769, p<0.05)$. Blood ethanol concentrations were measured weekly using tail blood samples collected $2 \mathrm{~h}$ after initiation of feeding and employing an Analox AM1 Alcohol Analyzer (Analox, Lunenburg, MA). After 30 days of treatment, all animals were returned to the ad libitum rat chow diet. AF animals underwent behavior studies during the $42 \mathrm{~h}$ after completion of alcohol feeding to assess whether the animals underwent alcohol withdrawal. Two weeks after returning to $\mathrm{AD}$ diet, estrous cycle changes in all animals were monitored by daily vaginal smearing. During their second estrous cycle on the day of proestrus (about three weeks after alcohol withdrawal), animals were housed with an untreated male rat for breeding. The presence of vaginal plug or sperm in vaginal smears denoted the first day of pregnancy. $\mathrm{AF}, \mathrm{PF}$, and $\mathrm{AD}$ rats were bred to produce male offspring (AFM, PFM, and ADM) and female offspring (AFF, PFF, and ADF). Litters of each dam were normalized to eight pups (kept both sexes), to reduce confounding variables from litter size. Maternal behaviors in dams were observed on postnatal day (PND) 1-6. Most of the pups were left with their birthmother until weaning on day 23 and then housed ingroup with same sex siblings until used for endocrine, molecular and behavioral studies. Cross-fostering was done with only a group of animals to check whether maternal care affects preconception alcohol-induced changes in corticosterone response to lipopolysaccharides (LPS). For this, we crossed two pups (one male and one female) from each of six $\mathrm{AF}$ and of six PF dams to six $\mathrm{AD}$ dams. The litter size of these $\mathrm{AD}$ dams were also maintained, like the rest of animals used in the study, to a number of eight using four of their own and four AF and PF dams. These pups were weaned at day 23 and used for LPS challenge response study on PND 60. In some non cross-fostered $\mathrm{AD}, \mathrm{AF}$ and $\mathrm{PF}$ pups, 5-azadeoxycytidine (AZA; $5 \mathrm{mg} / \mathrm{kg}$; Sigma-Aldrich, Ronkonkoma, NY) or saline was administered subcutaneously $(5 \mathrm{mg} / \mathrm{kg})$ in every other day between PND 1-5. These pups were weaned at day 23 and used for behavior, gene expression and methylation studies. At the end of the experiments, whole brains were collected and immediately frozen in dry ice. Prior to gene and protein measurements, whole hypothalamus, hippocampus, and amygdala were dissected from frozen brains using a rat brain atlas (Paxinos and Watson, 1982). Hypothalamus was dissected from the ventral part of a coronal section approximately Bregma -0.3 to $-3.3 \mathrm{~mm}$, amygdala from approximately Bregma -1.8 to Bregma $-3.3 \mathrm{~mm}$, and hippocampus was dissected from approximately Bregma -3.3 to $-5.3 \mathrm{~mm}$.

\section{Maternal Behavior in Alcohol-Exposed Dams}

Maternal behaviors in dams were observed every $3 \mathrm{~min}$ at PND 1-6 between 0930-1030 and 1700-1800 hours and the occurrence of the following behaviors were noted: whether the dam was physically on the pups, the occurrence of licking and grooming, and whether the dam was nursing pups and her nursing posture (arched back nursing, side nursing, or blanket nursing). Decreased time spent on pups, less licking and grooming, and less arched back nursing are indicators of poor maternal care in rats (Cameron et al, 2005).

\section{Anxiety-Like Behavior in Offspring}

Male and female offspring at PND 90 after birth were tested for baseline anxiety-like behavior in the open field (OF) and elevated plus mazes (EPM) as previously described (Logan et al, 2015). The EPM consisted of a standard plus maze with four arms (each $50 \mathrm{~cm}$ long). The $\mathrm{OF}$ consisted of a square field $(100 \mathrm{~cm} \times 100 \mathrm{~cm})$. Behavioral testing was conducted between 1000 and 1800 hours during the animals' light cycle in dim lighting conditions with the apparatus around 100 lux. Behavior was video-recorded and videos for the EPM test were scored with Stop Watch behavioral monitoring software (CBN; Emory University) by a scorer blind to the animal treatment group. For the OF, videos were tracked by AnyMaze (Stoelting; Wood Dale, IL) tracking software.

\section{Stress Hormone Responses to an Immune Challenge}

Adult male and female offspring were tested around days 60 after birth for stress hormone (corticosterone and adrenocorticotropic hormone, ACTH) responses to lipopolysaccharide (LPS). Because sex steroids affect corticosterone response to acute stress in rats, we used female rats at the stage of diestrus (determined by vaginal smearing) when estrogen and progesterone levels are maintained in a low concentration (Kalil et al, 2013). Also, to avoid circadian fluctuations of hormone levels, we conducted the experiment only in the late morning. Blood samples were collected from tail vein at $0 \mathrm{~h}$ (basal level) before and $2 \mathrm{~h}$ after (stimulated level) i.p injection of $100 \mu \mathrm{g} / \mathrm{kg}$ LPS. We used a 2 -h period to determine the stress hormone response, since plasma corticosterone and ACTH show their maximal responses at $2 \mathrm{~h}$ following the LPS treatment (Govorko et al, 2012). All samples were run in the corticosterone ELISA (IBL,Toronto, ON) and ACTH ELISA (MyBioSource, San Diego, CA) in duplicates.

\section{Measurement of Tissue Protein Levels}

Stress regulatory protein levels were measured in brain tissue samples using ELISAs. Frozen tissue samples were quickly removed and samples homogenized in a lysis buffer $(150 \mathrm{~mm}$ $\mathrm{NaCl}, 25 \mathrm{~mm}$ Tris, 1\% NP-40, 5\% glycerol, $1 \mathrm{~mm}$ PMSF, $10 \mu \mathrm{g} / \mathrm{ml}$ aprotinin, $1 \mu \mathrm{g} / \mathrm{ml}$ leupeptin and $0.5 \mathrm{~mm}$ sodium vanadate). Homogenates were centrifuged and the supernatants used for measurement by ELISA of tissue $\beta$-endorphin (Peninsula Laboratory, San Carlos, CA), CRF (Phoenix, Burlingame, CA) and arginine vasopressin (AVP) levels (MyBioSource, San Diego, CA). 


\section{Gene Expression Analysis by qRT-PCR}

Stress regulatory genes in various brain tissue samples were measured by qRT-PCR. Total RNA was isolated from tissue sample using RNeasy kit (Qiagen, Valencia, CA). The qRT-PCR conditions were as previously described (Govorko et al, 2012). The mRNA levels of corticotropin releasing factor (Crf), CRF receptor 1 (Crfrl), glucocorticoid receptor $(G r)$, mineralocorticoid receptor $(M r)$, proopiomelanocortin (Pomc), neuropeptide Y (Npy), Rpl-19, and Gapdh were measured using the primer sequences described in Supplementary Table S1. All runs were performed in duplicates. RT-PCR was performed using the ABI prism $7500 \mathrm{HT}$ sequence detection system (Applied Biosystems, Foster City, CA). Thermocycler conditions were $5 \mathrm{~min}$ at $95^{\circ} \mathrm{C}$, followed by 40 cycles of: $15 \mathrm{~s}$ at $95^{\circ} \mathrm{C}, 30 \mathrm{~s}$ at $60^{\circ} \mathrm{C}$, $40 \mathrm{~s}$ at $72^{\circ} \mathrm{C}$. For CRF-1, 45 cycles were used. Gene expression was compared with a standard curve prepared from control samples. We have previously shown that alcohol feeding similar to the procedures used in this study produced no significant effect on the expression of house keeping genes Gapdh, Rpl-19 and $\beta$-actin (Govorko et al, 2012). Similarly, in this study we found no effect of PCAE on Gapdh and Rpl-19 gene expression (Supplementary Fig. S1). Because of these, we feel Gapdh and Rpl-19 genes were stable during the alcoholfeeding paradigm. Hence, we presented the expression levels of hormones as ratios of both Gapdh and Rpl-19.

\section{DNA Methylation Analysis by Methylation Specific PCR (MSPCR)}

Genomic DNA was isolated by DNeasy Blood and Tissue Kit (Qiagen) from brain tissues. Samples were bisulfite treated using EZ DNA Methylation kit (Zymo, Irvine, CA). MSPCR was used to examine methylation status of the Crfr1 and Pomc genes as previously described (Govorko et al, 2012; Wang et al, 2013). MSPCR was not successful to detect Crf gene methylation. Primers specific for methylation/ unmethylation were designed using Methyl Primer Express program (Applied Biosystems; Supplmentary Table S1). qPCR was used to compare the amplification using methylation specific $v s$ unmethylation specific primers to obtain a ratio of methylation status. Prior to $\mathrm{qPCR}$, preamplification using the same conditions and by running samples for 12 rounds, and a $2 \mu$ l aliquot of each pre-amp sample was used for subsequent qPCR by sybr green in a total volume of $25 \mu \mathrm{l}$. All wells were loaded in duplicate. Thermocycler conditions were $5 \mathrm{~min}$ at $95^{\circ} \mathrm{C}$, followed by $40-45$ cycles of: $15 \mathrm{~s}$ at $95^{\circ} \mathrm{C}, 30 \mathrm{~s}$ at $60^{\circ} \mathrm{C}, 40 \mathrm{~s}$ at $72^{\circ} \mathrm{C}$. Standard curve methods were used for calculations of mean quantity.

\section{Bisulfite Pyrosequencing}

In order to characterize the extent of cytosine methylation of CpG dinucleotides in the Crf, and Pomc proximal promoter, we designed bisulfite sequencing primers in the CpG island. Bisulfite pyrosequencing was not successful to detect Crfr1 gene methylation. The methods for Crf and Pomc bisulfite pyrosequencing were as described previously (Govorko et al, 2012; Chen et al, 2012). A MethPrimer program was used to assess the $\mathrm{CpG}$ island and bisulfite sequencing primers for these three genes (Supplementary Table S1). Following bisulfite treatment, PCR reaction was performed with bisulfite sequencing primers using Pyromark reagents (Qiagen, Valencia, CA) as detailed in manufacturer's protocols. Pyrosequencing was carried out using a sequencing primer on a PSQ-HS-96A model pyrosequencer (Qiagen). In the study we analysed one control $\mathrm{C}$ in non $\mathrm{CpG}$ background for efficient $\mathrm{C} \rightarrow \mathrm{T}$ conversion. Each $\mathrm{CpG}$ in the study was analysed by comparing $\mathrm{C} / \mathrm{T}$ peaks. The percentage of methylation was represented using percent $\mathrm{C}$ remaining as $\mathrm{C}$ in each target $\mathrm{CpG}$.

\section{Statistical Analysis}

The data shown in the figures and text are mean \pm SEM. Comparisons between groups were made using one-way analysis of variance (ANOVA) with post hoc analysis using the Newman Keuls posttest. Two-way ANOVA with Bonferroni post hoc tests were used to correct for multiple comparisons. Significance was set at $\alpha<0.05$. F-statistics and $P$ values of major data (Figures $1,2,3,4,5$ ) are shown in Supplementary Table S2.

\section{RESULTS}

Preconception Alcohol Drinking Affects Pregnancy
Outcomes without Altering Maternal Care Behaviors

Adult cyclic female rats when given free access to $6.7 \%$ alcohol containing liquid diet and drinking water, frequently drank alcohol to achieve a blood alcohol level between 0.08 and $0.13 \mathrm{~g} / \mathrm{dl}$ (Figure 1a). After 4 weeks of alcohol use, when AF rats were restricted from any alcohol they showed mild alcohol withdrawal symptoms (rigidity and agitation but no tremor or convulsion) lasting only 16 to $24 \mathrm{~h}$ (Supplementary Table S3). After feeding the animals a regular diet for a period of 2 weeks, the body weight of these animals were measured, and no detrimental effect of prior alcohol use on body weight was noted (Figure 1b; AF animals showed a slight but not significant increase in weight). The $\mathrm{AF}$ animals, like the control (PF and AD) animals, showed a normal 4-day estrous cycle (Figure 1c). However, when the AF rats were bred, about three weeks after alcohol discontinuation, they had longer gestation time in days (Figure 1d) and moderately smaller sized litters (Figure 1e). The sex ratio in litters did not differ between the treatment groups (Figure 1f and g), but the mean litter birth weight of $\mathrm{AF}$ dams was lower than those of $\mathrm{AD}$ and $\mathrm{PF}$ dams (Figure $1 \mathrm{~h}$ and i). Inspection of body growth weekly during the developmental period revealed lower body weight in male and female offspring of AF dams, AFM and AFF rats, respectively (Figure $1 \mathrm{j}$ and $\mathrm{k}$ ). When tested for maternal care behaviors, no differences in time spent on pups, licking and grooming, and arched back nursing were noted in AF, PF and $\mathrm{AD}$ dams (Supplementary Figure S2).

\section{Preconception Alcohol Exposures (PCAE) Alter the Hypothalamic-Pituitary-Adrenal (HPA) Axis Functions and Anxiety-Like Behaviors}

To investigate whether preconception alcohol feeding affected the stress axis response, we first measured the basal and LPS-induced plasma corticosterone levels. This test 
a

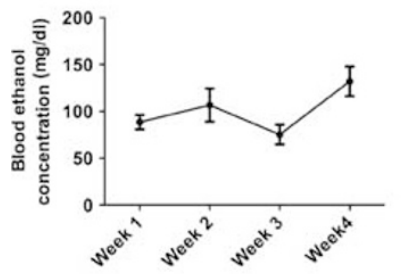

d

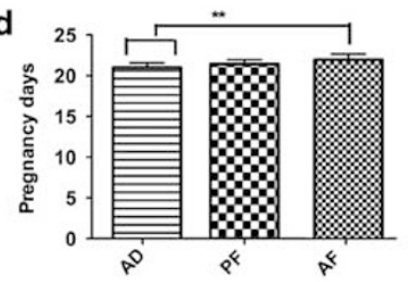

g

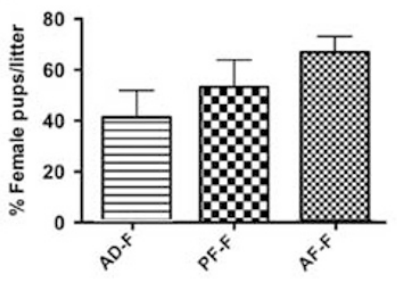

b
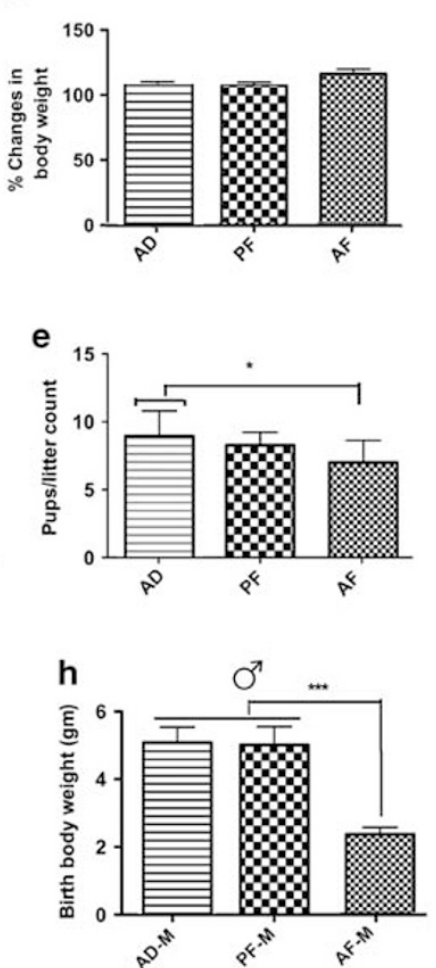
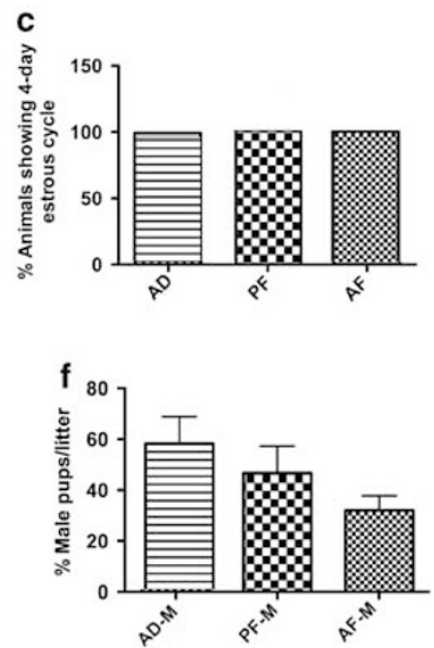

i

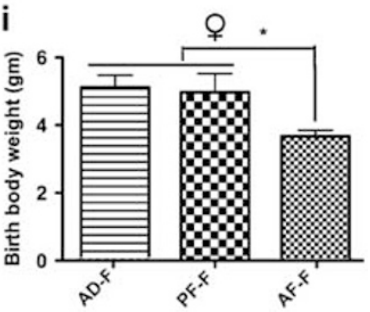

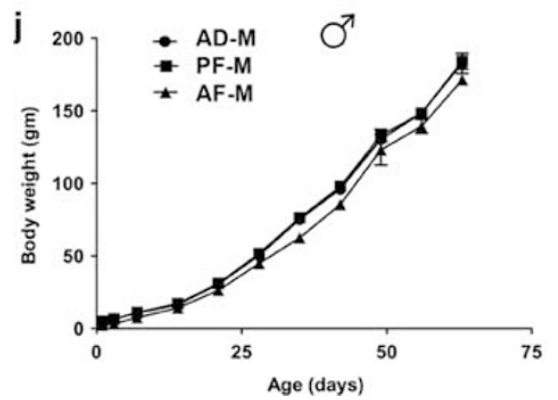

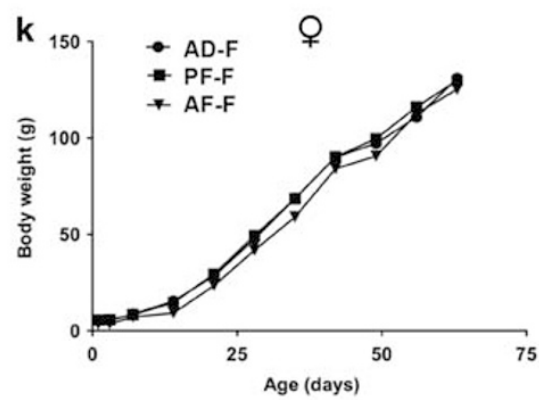

Figure I Effects of preconception alcohol feeding on pregnancy outcomes. Physiological parameters and pregnancy outcomes of preconception alcohol feed rats are shown. Time-dependent changes in blood concentrations of ethanol in female rats who had free access to alcohol in liquid diet for a 4-week period and were used in preconception alcohol feeding study (a). Changes in body weight before and two weeks after ethanol withdrawal (b). Estrous cycle patterns two weeks after ethanol withdrawal (c). Pregnancy outcome of the alcohol-fed rats (AF) were compared with those of pair-fed (PF) and ad lib-fed (AD) control rats by pregnancy days (d), total pups/litter count (e), \% male or female pups/litter count ( $f$ and g), and birth body weight of male or female offspring pups ( $h$ and i). Data are mean $\pm \operatorname{SEM}(n=7)$ and were compared by one-way analysis of variance (ANOVA) and Newman-Keuls post-test. * $p<0.05$, *** $p<0.01$, **** $p<0.00 \mathrm{I}$, AF vs PF and AD. The body growth curves of male offspring (AF-M, PF-M, and AD-M) or female offspring (AF-F, PF-F, and AD-F) of dams fed with different diets (AF, PF, and $A D$ ) were shown in panels $j$ and $k$, respectively. Data were analyzed by two-way ANOVA (treatment $\times$ time) followed by the Bonferroni post-test. A significant time $\times$ treatment effect on body growth was noted for both male $(p<0.00 \mathrm{I})$ and female offspring $(p<0.00 \mathrm{I})$.

revealed a moderate increase (not significant) in basal corticosterone levels, but a marked increase in corticosterone response to the immune challenge in both male (AFM; Figure 2a) and female offspring (AFF; Figure 2b) of AF dams. To check if the higher corticosterone response to the immune challenge in PCAE offspring might be related to altered nursing behaviors of AF dams, we determined the corticosterone response to LPS in AFM and AFF rats who were fostered to control (AD) dams during the nursing period. These data indicate that both AFM and AFF offspring who were fostered also had higher corticosterone response to the LPS challenge, suggesting the effect of PCAE was not related to altered maternal care by AF dams (Supplementary Figure S3A and B). Hence, we used the male and female offspring of $\mathrm{AF}, \mathrm{PF}$ and $\mathrm{AD}$ dams without any fostering in further characterization of the HPA axis functions.

We measured plasma adrenocorticotropin hormone (ACTH), a corticosterone-regulatory pituitary hormone, and the levels of ACTH regulatory hypothalamic hormones $\mathrm{CRF}, \mathrm{AVP}$, and $\beta$-endorphin in male and female offspring of $\mathrm{AF}, \mathrm{PF}$, and AD dams. These tests revealed that the ACTH response to LPS challenge was higher in AF-M and AF-F rats than those in PF-M and PF-F or AD-M and AD-F rats 

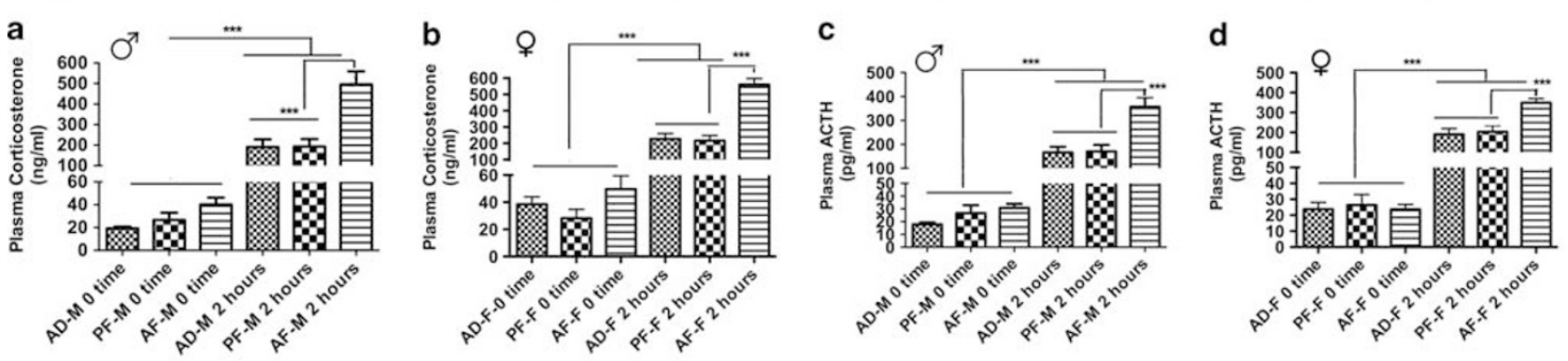

Hypothalamic hormones
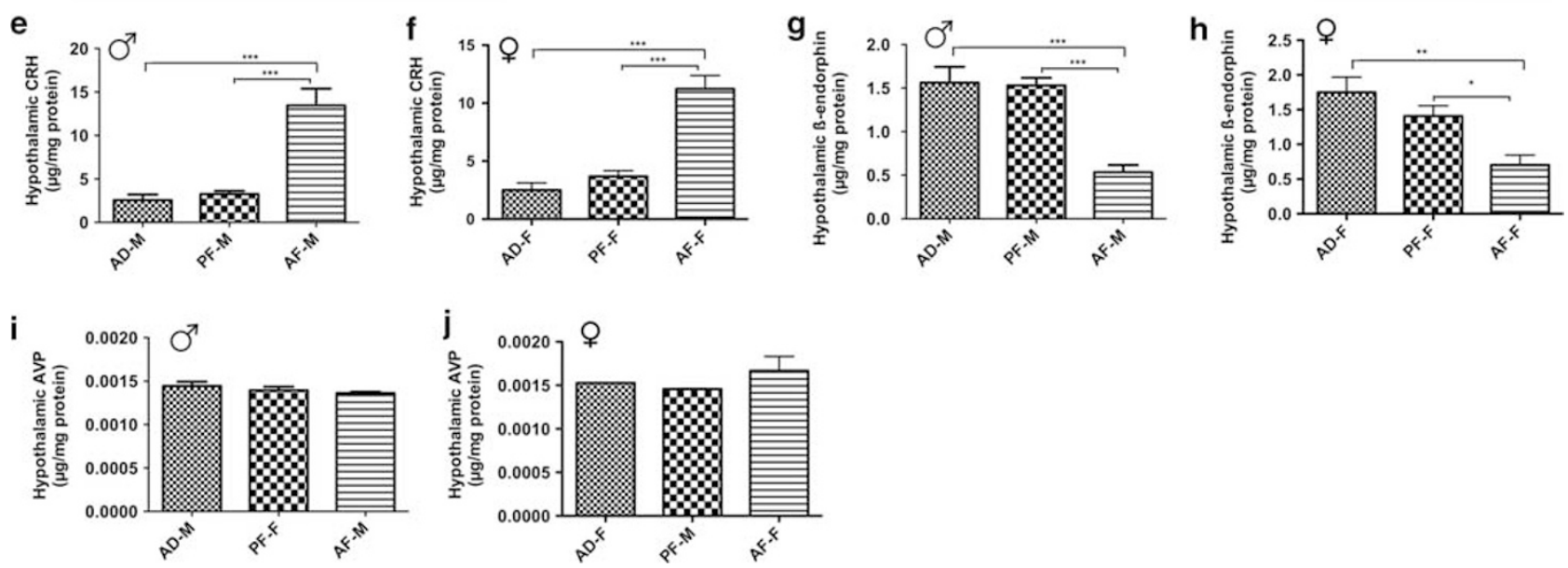

Figure 2 Consequences of preconception alcohol feeding on stress axis responses in adult male and female offspring. The stress axis function tests include measurement of plasma levels of corticosterone $(\mathrm{a}, \mathrm{b})$ and ACTH (c, d) measured before ( 0 time) and $2 \mathrm{~h}$ after LPS injection and the basal hypothalamic protein level of CRF (e, f), $\beta$-endorphin $(g, h)$, and AVP $(i, j)$ in adult male offspring (AF-M, PF-M, AD-M) or female offspring (AF-F, PF-F, AD-F) of dams fed with different diets (AF, PF and AD). Data are mean \pm SEM $(n=6)$. Data were analyzed using one-way ANOVA followed by the Newman-Keuls post-test. $* p<0.05$, $* * * 0.01$, **** $p<0.00$ I, AF (both M and F) vs AD and PF (both M and F) groups. (a) $P<0.00$ I, within treatment group-corticosterone levels at 0 time vs $2 \mathrm{~h}$ post LPS.

(Figure 2c and d). Both AF-M and AF-F groups also showed higher levels of CRF (Figure 2e and f), but showed lower levels of $\beta$-endorphin in the hypothalamus compared with control groups (PF-M, PF-F, AD-M, AD-F; Figure $2 \mathrm{~g}$ and $\mathrm{h}$ ). Hypothalamic AVP levels between groups did not differ (Figure $2 \mathrm{i}$ and $\mathrm{j}$ ). No sex differences in PCAE effects on hypothalamic peptide contents were noted.

\section{PCAE Effects on Anxiety-Like Behavior}

Next, we tested the baseline anxiety-like behaviors of PCAE animals and control offspring in the OF and EPM. We show here that the male AF-M rats showed significantly less time spent in the center zone of the OF as compared with $\mathrm{AD}-\mathrm{M}$ rats, although comparisons between $\mathrm{AD}-\mathrm{M}$ and $\mathrm{PF}-\mathrm{M}$ were not significantly different (Figure $3 \mathrm{a}$ ). For females (Figure $3 \mathrm{~b}$ ), the time spent in the center zone in the OF by AF-F animals did not differ from those in PF-F and AD-F rats. AF-M rats spent significantly less time in the open arms of the EPM compared with AD-M, but not PF-M, rats (Figure $3 \mathrm{c}$ ). In addition, male $\mathrm{AF}$ and $\mathrm{PF}$ offspring made less entries into the open arms of the EPM compared with $\mathrm{AD}$ males (Figure 3d). In males, there were no significant differences between groups in total arm entries (Figure 3e), a measure of overall locomotor behavior in this test. For female AF-F, PF-F, and AD-F rats, there were no significant differences in either time spent in the open arms of the EPM (Figure 3f), entries into the open arms of the EPM (Figure $3 \mathrm{~g}$ ), or total arm entries (Figure $3 \mathrm{~h}$ ).

\section{PCAE Effects on the Stress Regulatory Genes}

To understand the cause for the elevated HPA axis functions and increased anxiety-like behaviors in PCAE offspring, we measured various regulatory genes (Crf, Crfrl, Gr, Mr, Pomc, and $N p y$ ) in the hypothalamus and/or the limbic system in these and control animals. Because we did not find sex differences in the HPA axis function and we only saw anxiety-related behavioral changes in male offspring, we focused our stress regulatory gene study only in males. We also did not measure $A v p$ mRNA levels since its protein level did not change following PCAE. Measuring brain region specific changes in the regulatory genes in PCAE offspring, we found the level of Crf mRNA in AF-M animals was elevated in the hypothalamus (Figure $4 \mathrm{a}$ and $\mathrm{b}$ ) but not in hippocampus (Supplementary Figure S4A and B) or amygdala (Supplementary Figure S4C and D), as compared with AD-M and PF-M animals. Crfr1 mRNA levels in AF-M animals were elevated in hippocampus (Figure $4 \mathrm{c}$ and d) and amygdala (Figure 4e and $\mathrm{f}$ ) but not in the hypothalamus 


\section{OF test}

a

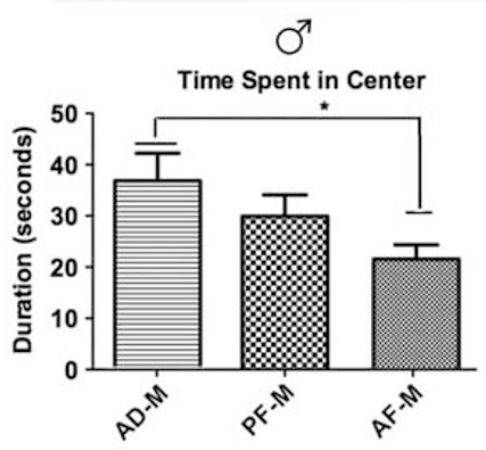

b
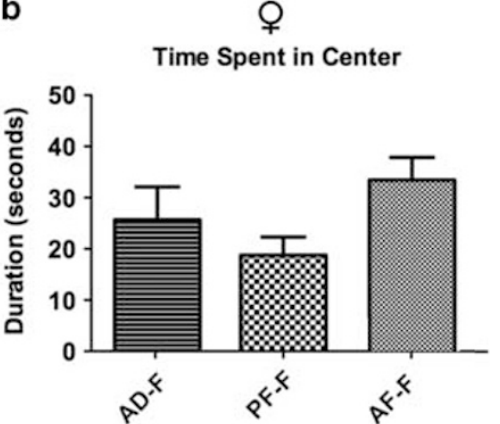

EPM test

c
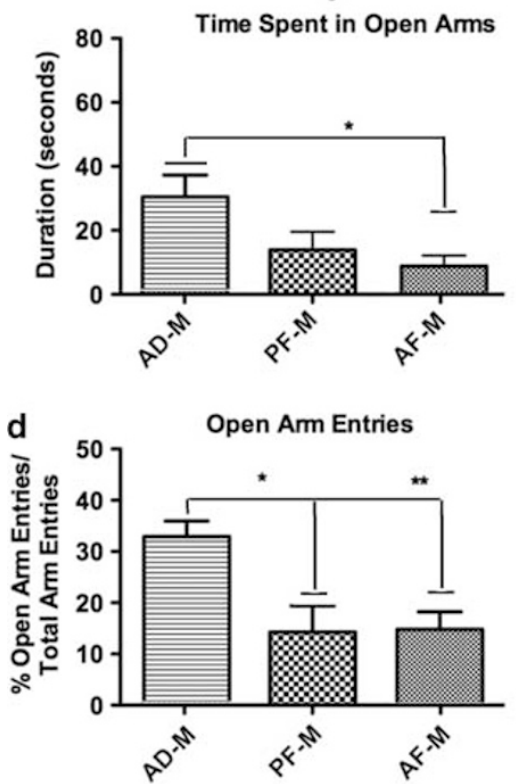

e

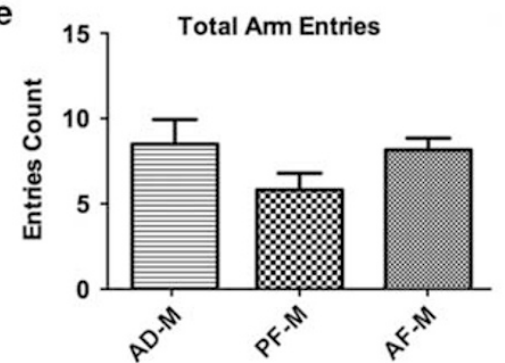

f
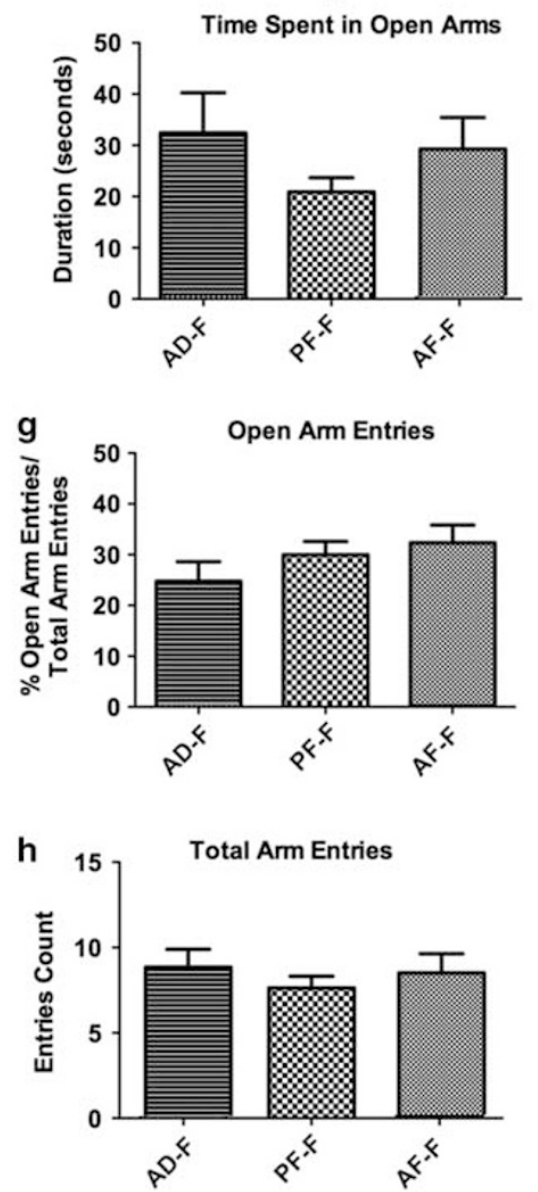

Figure 3 Consequences of preconception alcohol feeding on anxiety-like behaviors in adult male and female offspring. Adult AF-M rats spent less time in the center of the OF compared with AD-M offspring (a; * $p<0.05$ ) indicating increased anxiety-like behavior in AF-M offspring. AF-F rats did not differ from PF-F and AD-F in time spent in the center zone ( $b ; p>0.05)$. AF-M also showed significantly more anxiety-like behavior in the EPM, since they spent significantly less time in the open arms compared with AD-M (c) and made less entries into the open arms compared with AD-M (d). In males, there were no significant differences between groups in total arm entries (e). There were no significant differences between AF-F, PF-F, and AD-F females in anxiety-like behavior in the EPM $(f, g, h)$. Data are mean \pm SEM $(n=7-14 ; A D-M=9, P F M=7 ; A F M=14)$ and were analyzed using one-way ANOVA and NewmanKeuls post-test. $* p<0.05$, ** $p<0.01$, compared with the AD group.

(Supplementary Figure S4E and F). The Pomc mRNA level in the hypothalamus was found to be reduced in AF-M animals compared with AD-M and PF-M animals (Figure $4 \mathrm{~g}$ and $\mathrm{h}$ ). Pomc mRNA level in the hippocampus and amygdala were not determined since their roles in the HPA regulation have not been demonstrated. The mRNA levels of $G r, M r$ and $N p y$ in various brain areas were similar in AF-M, AD-M and PF-M animals (Supplementary Figure S4G-T). 


\section{PCAE Affects the Methylation Status of CpG Islands in Stress Regulatory Genes}

We tested whether promoter methylation status of genes are affected by the PCAE treatment. Both MSPCR assays and pyrosequencing procedures were used to examine the methylation status of HPA regulatory genes. We found methylation levels at different epigenetic sites of the Crf gene varied and PCAE moderately suppressed methylation of the CpG dinucleotides at -232 in the proximal promoter of Crf in the hypothalamus (Figure 4i). In addition, we found marked reduction in Crfrl methylation in AF-M animals in the hippocampus (Figure $4 \mathrm{j}$ ) and in the amygdala tissues (Figure 4k). However, we observed increased methylation of the Pomc gene in the hypothalamus of AF$\mathrm{M}$ offspring as compared with $\mathrm{AD}-\mathrm{M}$ and $\mathrm{PF}-\mathrm{M}$ offspring (Figure $4 \mathrm{l}$ and $\mathrm{m}$ ).

\section{Administration of a DNA Methylation Blocker Normalizes Stress Response and Anxiety-Like Behaviors}

To further determine the role of epigenetic programming, we tested the effects of AZA, a DNA methylation blocker (22) on PCAE-associated changes in the stress response, anxiety-like behavior and gene expression levels. For the stress test, we found increased ACTH and corticosterone responses following LPS challenge in saline-treated AF-F animals as compared with those in saline-treated PF-F and AD-F animals, whereas LPS-induced ACTH and corticosterone levels in AZA-treated AF-F animals were not different from those in AZA-treated AD-F and PF-F animals (Figure 5a-d). In the case of anxiety-like behavior in the OF test, we found significant effects of AZA. AF-M animals, when treated with saline, spent a reduced amount of time in the center zone compared with AD-M but not PF-M animals, but they spent a similar amount of time in the center as $\mathrm{AD}-\mathrm{M}$ and PF-M controls when treated with AZA (Figure 5e). In the EPM test, saline, but not AZA-treated AF-M rats, spent significantly less time in the open arms of the EPM compared with similarly treated $A D$ offspring (Figure 5f). In addition, saline- but not AZA-treated AF-M and PF-M, made less entries into the open arms of the EPM compared with similarly treated AD-M rats (Figure 5g). Nonetheless, comparison between AZA-treated AF, PF and $A D$ animals showed no differences in the entries into the open arms indicating AZA treatment normalized EPM behavior. For the gene expression measurements, we also found that AZA was effective in preventing PCAE induced changes in $\mathrm{Crf}$ (Figure $5 \mathrm{i}$ and $\mathrm{j}$ ) and Pomc gene expression (Figure $5 \mathrm{~m}$ and $\mathrm{n}$ ) in the hypothalamus and normalized the PCAE-induced changes in Crfr1 levels in the amygdala (Figure $5 \mathrm{q}$ and $\mathrm{r}$ ). For methylation study, we found that Aza treatment reduced methylation in all genes in all groups studied (Figure 5k, l, o, p and s). It is interesting to note that the effect of AZA on expression seems to be only on hypermethylated promoters as it was observed with Crf in control samples (Figure 5i and $\mathrm{j}$ ) and with Pomc in AF samples which have reduced expression (Figure $5 \mathrm{~m}$ and $\mathrm{n}$ ). Aza treatment did not elicit any increased expression of Pomc in control samples and Crf in AF samples even they have reduced level of methylation.

\section{DISCUSSION}

In the present study, we demonstrate chronic alcohol exposure in female rats that elevates blood alcohol level equivalent to human binge drinking level (NIAAA, 2016) for a period of four weeks produces no effect on the reproductive cycle or body weight after 3 weeks of alcohol withdrawal but significantly affects the pregnancy outcomes including longer gestation time, smaller size litters and lower birth weight of the litter. In addition, despite these PCAE dams showing normal maternal care behaviors their litter show reduced body growth throughout the nursing period.

Data presented here show that both male and female PCAE offspring had increased corticosterone and ACTH responses to the LPS challenge during the adult period. Interestingly, similar stress hyperresponse to the LPS challenge has been reported in fetal alcohol exposed rat offspring (Govorko et al, 2012). This identifies a similarity between PCAE offspring and fetal alcohol exposed offspring. The higher stress hormone response to the immune challenge in adulthood in PCAE offspring persisted even after maintaining them under the maternal care of a control foster dam, suggesting the possibility that the stress abnormality is not caused by altered maternal care but possibly due to inheritance of alcohol marks influencing stress axis functions.

We also show male PCAE offspring spend significantly less time in the center zone of the OF and in the open arms of the EPM compared with AD controls, indicating increased anxiety-like behavior in PCAE males. Notably, effects of the maternal pair feeding (PF) condition on male offspring were also observed in the behavior studies, as PF males showed increased anxiety in the EPM compared with AD males and a modest trend for increased anxiety in the OF. The PF condition is included in alcohol-exposure studies as a control for diet and handling factors involved in alcohol administration. Thus, we cannot say conclusively that the anxietylike behavior observed in AF males is due specifically to

\footnotetext{
Figure 4 Effects of preconception alcohol drinking on expression and methylation of stress regulatory genes in various brain areas. Adult AF-M rats showed tissue specific changes in mRNA expression, as determined by q-RT-PCR, of Crf (a, b), Crfrl (c-f) and Pomc (g, h) in the hypothalamus, hippocampus, and/or amygdala. AF-M rats had increased Crf mRNA compared with PF-M and AD-M rats in the hypothalamus (a, b). In addition, AF-M rats had increased Crfr I mRNA compared with PF-M and AD-M rats in the hippocampus (c, d) and amygdala (e, f). Pomc mRNA levels in the hypothalamus were lower in AF-M compared with AD-M and PF-M offspring ( $g, h)$. AF-M rats also showed differential methylation patterns of Crf (i), Crfrl in hippocampus (j) and amygdala ( $k$ ) and Pomc (I, m) genes in hypothalamus. AF-M rats had suppressed CRF methylation in the hypothalamus (i) of the CpG dinucleotides at - 232 in the proximal promoter as determined by pyrosequencing methods. AF-M rats also showed a reduction in Crfrl methylation in the hippocampus (j) and amygdala ( $k$ ) as determined by MSPR assay. In contrast, the methylation of the POMC gene was increased in the hypothalamus of AF-M as compared with AD-M and PF-M $(\mathrm{I}, \mathrm{m})$ as determined by MSPR assay and or pyrosequencing assay. Data are mean $\pm \mathrm{SEM}(n=7$, except $n=12$ in AF group shown in i) and were compared by a one-way ANOVA followed by the Newman-Keuls post-test. ${ }^{*} p<0.05$, $* * * 0.0$ I, **** $p<0.00$ I, group with this symbol vs AF-M.
} 
PCAE, as the PF condition also had a moderate anxiogenic effect, and the effects of preconception maternal diet and stress may have contributed to the observed increases in anxiety-like behavior in male AF offspring. However, it is also a possibility that the overlapping behavioral effects observed in AF and PF male offspring do stem from distinct
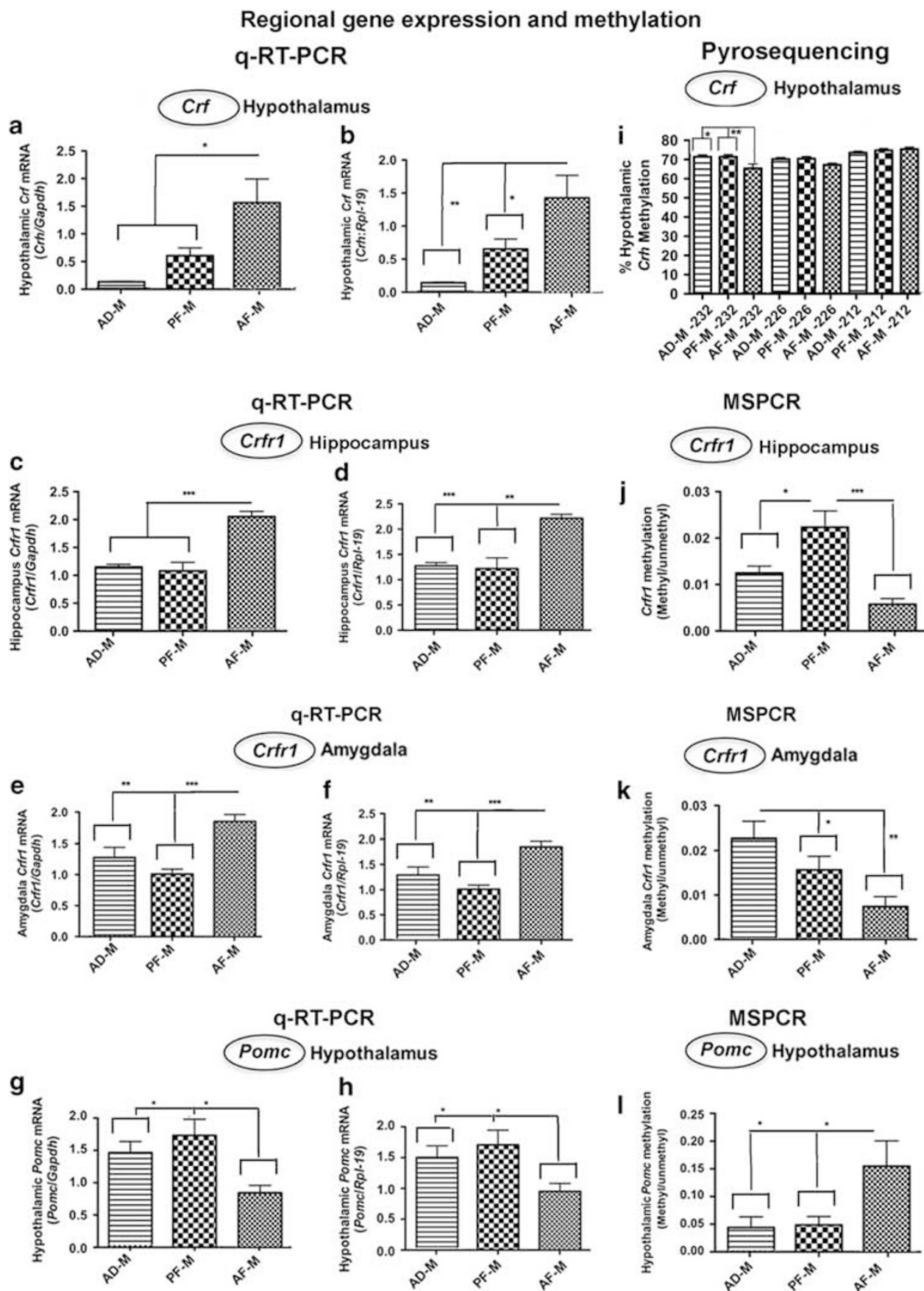

q-RT-PCR

Crfr1 Amygdala

\section{Pyrosequencing}

Crf Hypothalamus

MSPCR

Crfr1 Hippocampus
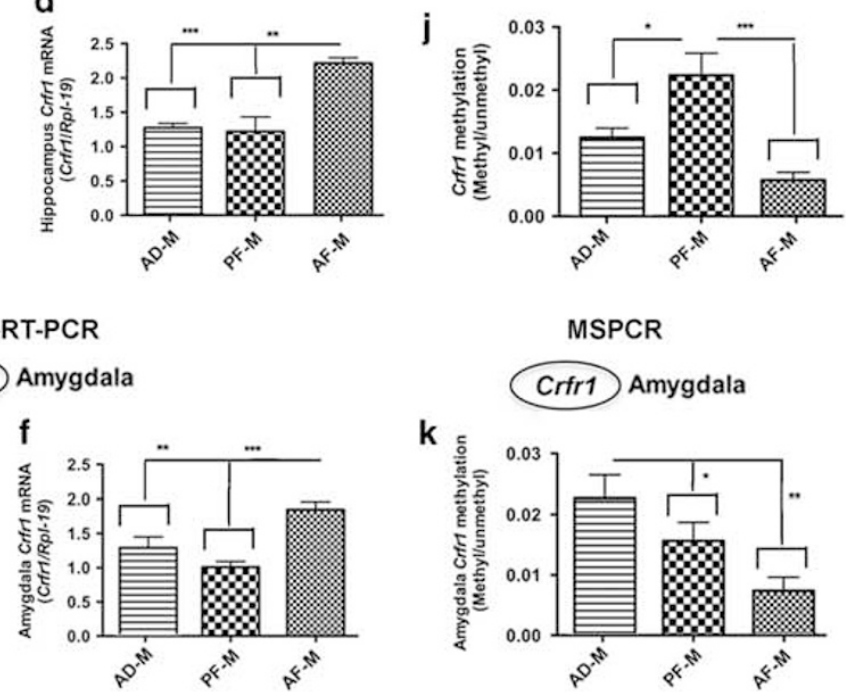

MSPCR
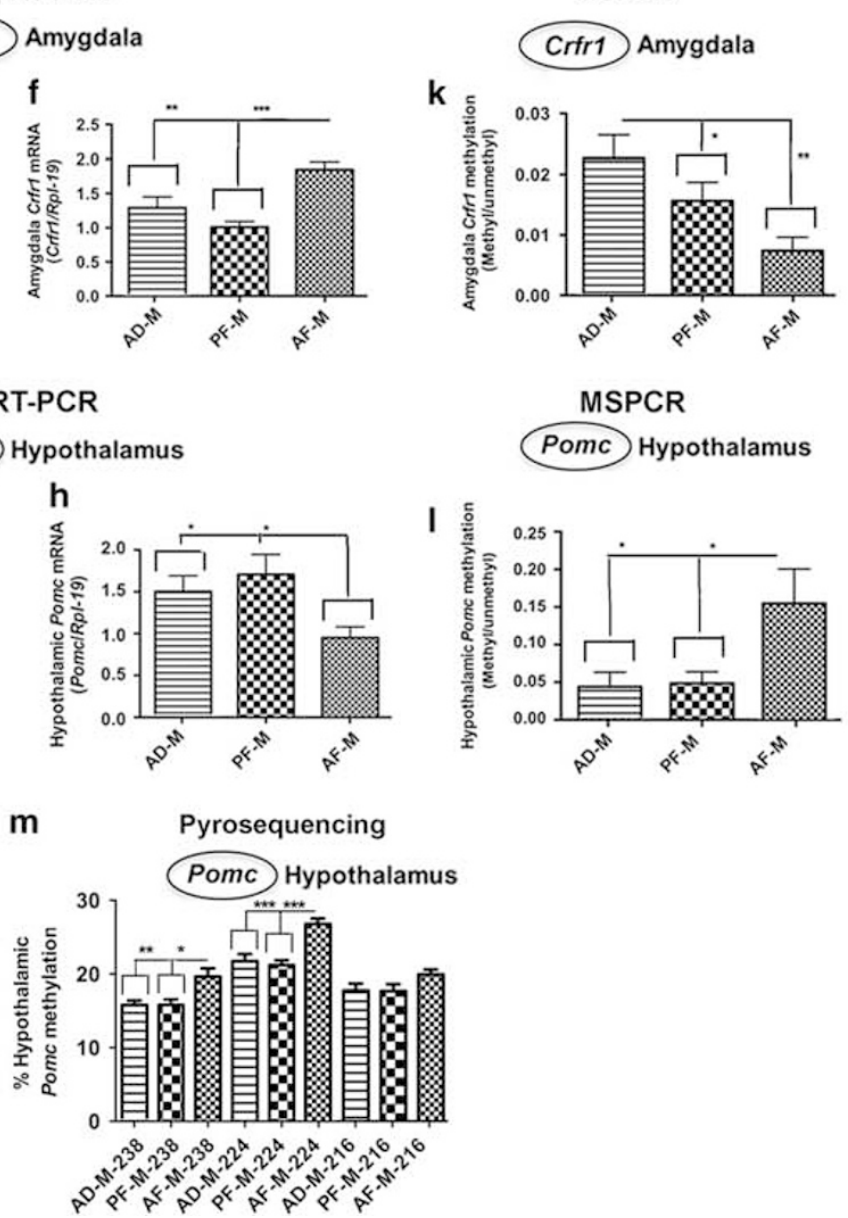
causes, but manifest in a similar manner in behavior. Similar to our findings, other studies utilizing pair feeding or sham pair feeding controls in fetal alcohol exposure models have observed effects of the PF conditions on both the HPA axis and the neuroimmune system that overlap with the effects observed in AF conditions (Boschen et al, 2016; Glavas et al, 2007). In addition, preconception stress and diet alterations have been shown to affect offspring (Leshem and Schulkin, 2012; Li et al, 2010; McGowan et al, 2008). However, there was no effect of the PF condition on other measures of stress vulnerability, including stress-related gene expression, protein levels, immune stress response, or gene methylation (discussed below), as PF animals did not differ from $\mathrm{AD}$ control animals in these studies. Thus, the driving force for the majority of phenotypes observed in our studies can be concluded to be due specifically to preconception maternal alcohol, and not due to other factors such as maternal stress or nutrition.

Interestingly, both male and female AF offspring show increased CORT and ACTH levels in response to LPS, whereas, only male AF offspring, and PF offspring to some extent, show increased anxiety behavior. The reason for the observed sex differences in behavior are likely due to inherent sex differences in rats in these behavioral tests, as female rats, in comparison with males, have been shown to be less susceptible to anxiety behavior in the EPM and OF tests (Blizard et al, 1975; Osborn et al, 1998; Johnston \& File, 1991). Thus, it is possible that these behavioral tests were not sufficiently stressful enough to assay anxiety-like behavior in females, and future studies utilizing different behavioral measures of anxiety are needed to explore the behavioral effects of PCAE in females. Similarly, fetal alcohol exposure studies have found sex differences on the effects of prenatal alcohol exposure on behavior, with males being more susceptible to fetal-alcohol-induced anxiety and depression in certain behavioral tests (Hellemans et al, 2010). Moreover, although increased corticosterone signaling is associated with increased anxiety-like behavior in the EPM and OF, behavior in these tests are likely a manifestation of effects on multiple hormonal and neural substrates. The OF and EPM tests are also known to be heavily regulated by the GABAergic and serotonergic systems (Osborn et al, 1998; Tu et al, 2014), which were not investigated in these studies and might be explored in future studies using our PCAE model. Finally, another factor in the observed sex difference in behavior might be the modulating effects of ovarian hormones on behavioral testing, as, in the LPS study, females were only used in diestrus, whereas in the behavior studies, estrus cycle was not restricted to diestrus, and some females were likely in estrus and proestrus during testing. It is possible, variations in ovarian hormones may have altered the effects of preconception alcohol on behavior in female offspring. For these reasons, the effects of preconception alcohol on hormonal response to stress and anxiety-like behavior might be divergent, as was observed in our studies.

Enhanced ACTH and corticosterone responses to stress in rats are known to result from increased CRF and/or AVP levels in the hypothalamus and possibly by impaired negative-feedback control by GR, MR and CRFR1 receptors in the limbic areas and by the POMC-derived peptide $\beta$-endorphin in the hypothalamus that functions to terminate stress responses (McEwen et al, 2015; Sarkar and Zhang,
2013). In addition, anxiety-related behavior is regulated by Crfr1 in the amygdala (Gilpin et al, 2015) and NPY in the hypothalamus and limbic areas (Sotnikov et al, 2014). The data of the present study show that in PCAE offspring increased levels of hypothalamic CRF and reduced levels of hypothalamic POMC/ $\beta$-endorphin; identifying a dysregulation in the functions of two key regulatory neurotransmitters of the HPA axis. PCAE offspring also showed increased levels of Crfr1 mRNA levels in the amygdala and hippocampus. Thus, PCAE offspring have significant alterations in the neuroendocrine system regulating HPA axis function.

DNA methylation is known to inhibit gene transcription by interfering with the recruitment of transcription factors or by recruiting methylated-DNA binding proteins that decrease transcription efficiency (Vaissière et al, 2008). We showed here that PCAE induces long-lasting hypermethylation of the Pomc gene, while causing hypomethylation of Crf and Crfrl genes. This inference is based on the findings that the percentage of the cytosine methylation of CpG-rich sites adjacent to the gene transcription start site for Pomc was higher, whereas for Crf and Crfrl was lower in PCAE animals than in controls. Methylation of the proximal region of the promoter often correlates closely with a negative effect on gene expression (Vaissière et al, 2008). Moreover, deletions or mutations in these promoter regions cause a significant decrease in gene expression for Pomc, Crf and Crfr1 (Chen et al, 2012; Sotniko et al, 2014; Ehrlich et al, 2010). Hence, increased DNA methylation in the key epigenetic island in the promoter regions of the Pomc gene could have impaired the function of the hypothalamus resulting in reduced expression levels of this gene, as has been demonstrated in the PCAE offspring. Conversely, reduced DNA methylation in the proximal region of $\mathrm{Crf}$ and Crfr1 promoters in key HPA regulatory brain tissues could have resulted in the increased expression levels of these genes in PCAE offspring.

We further confirm the role of promoter methylation in suppressing the gene expression as well as altered behavior by treating the rat offspring with AZA, a blocker of the regulators of gene promoter methylation, DNA methyltransferases (Vaissière et al, 2008). PCAE saline-treated animals, and PF saline-treated animals to some extent, showed increased anxiety-like behavior in the EPM and OF compared with AD offspring. However, comparison between AZA-treated AF, PF and AD animals showed no differences in behavior, indicating AZA treatment normalized anxietylike behavior. Aza treatment also reduced the promoter methylation of Pomc and Crf in the hypothalamus. Aza treatment normalized Pomc expression in hypothalamus of AF rat offspring, whereas Aza treatment increased the expression of Crf in control rats but did not produce any effect in AF rats. These results show although Aza reduces methylation, its effect on expression seems to be specific to only hypermethylated promoters as it was observed with Crf in control samples and with Pomc in AF samples, which have reduced expression. Aza treatment did not elicit any increased expression of Pomc in control samples and Crf in AF samples as they have reduced levels of methylation. We and others previously reported that hypermethylated promoters recruit methyl $\mathrm{CpG}$ binding proteins to repress the transcription of Pomc and tumor suppressor genes 
Stress-normalization by a methylation blocker
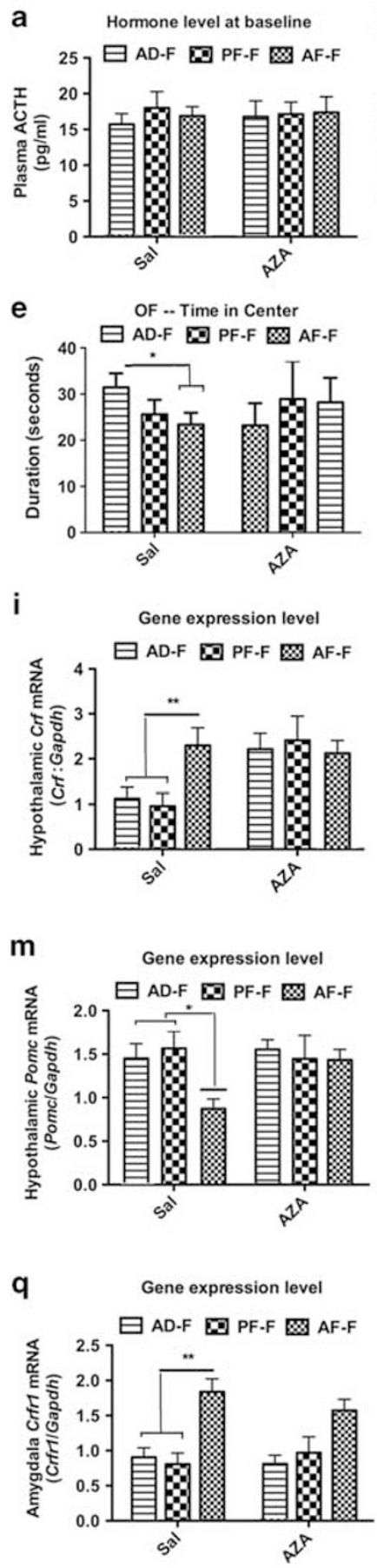
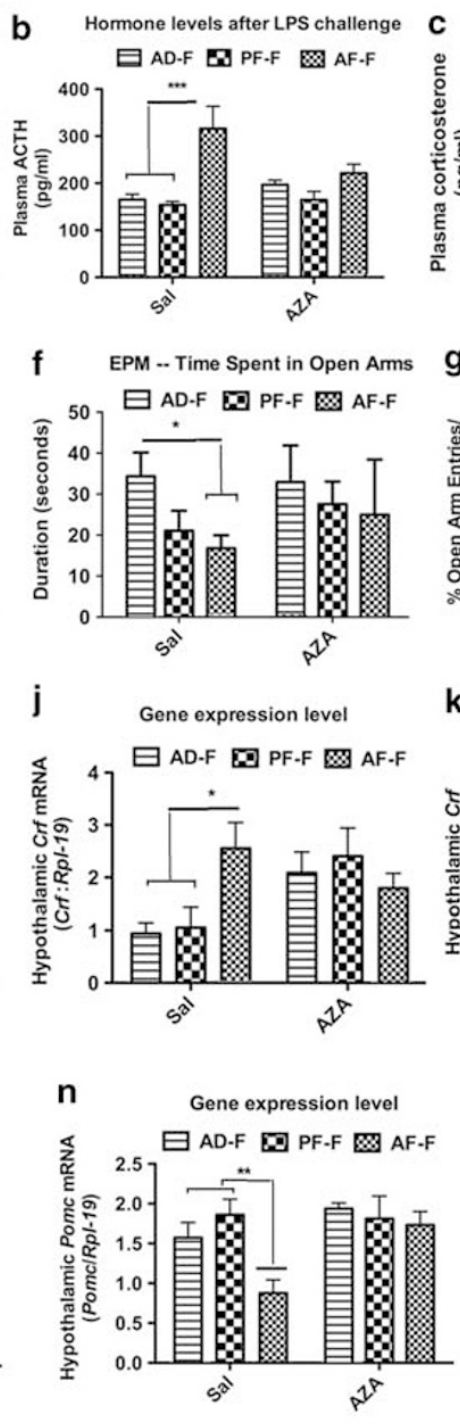

r

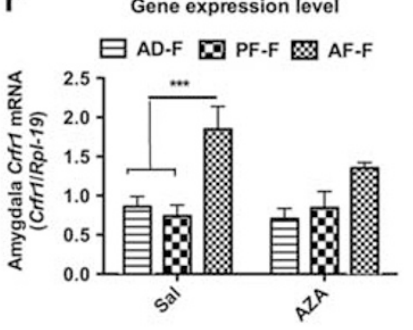

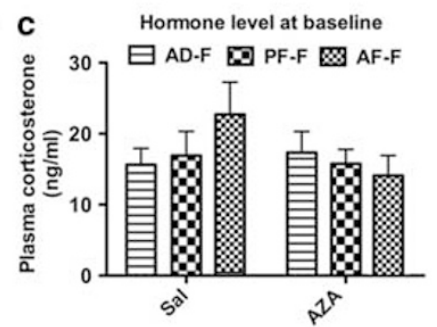
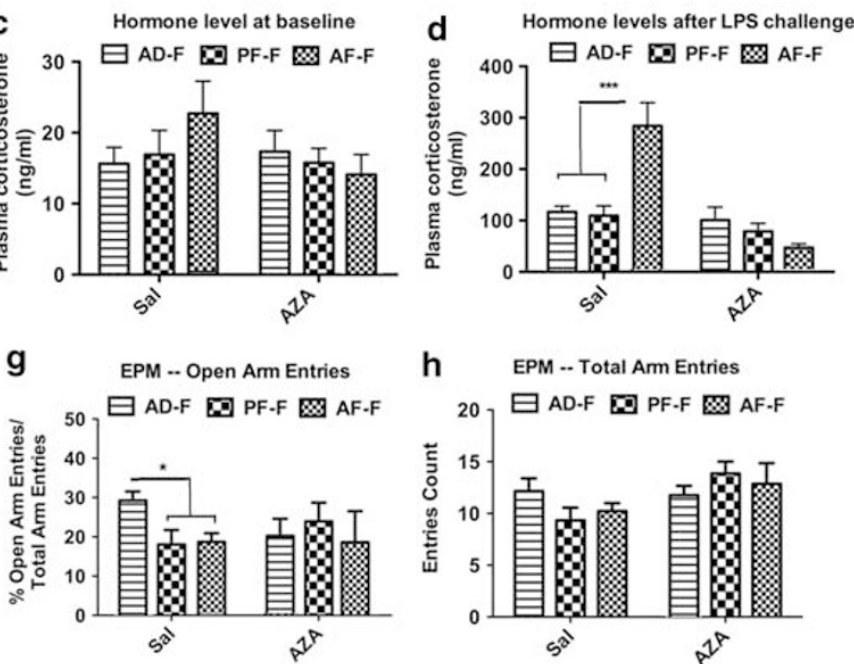

h EPM -- Total Arm Entries
20 日 AD-F PF-F $\otimes$ AF-F
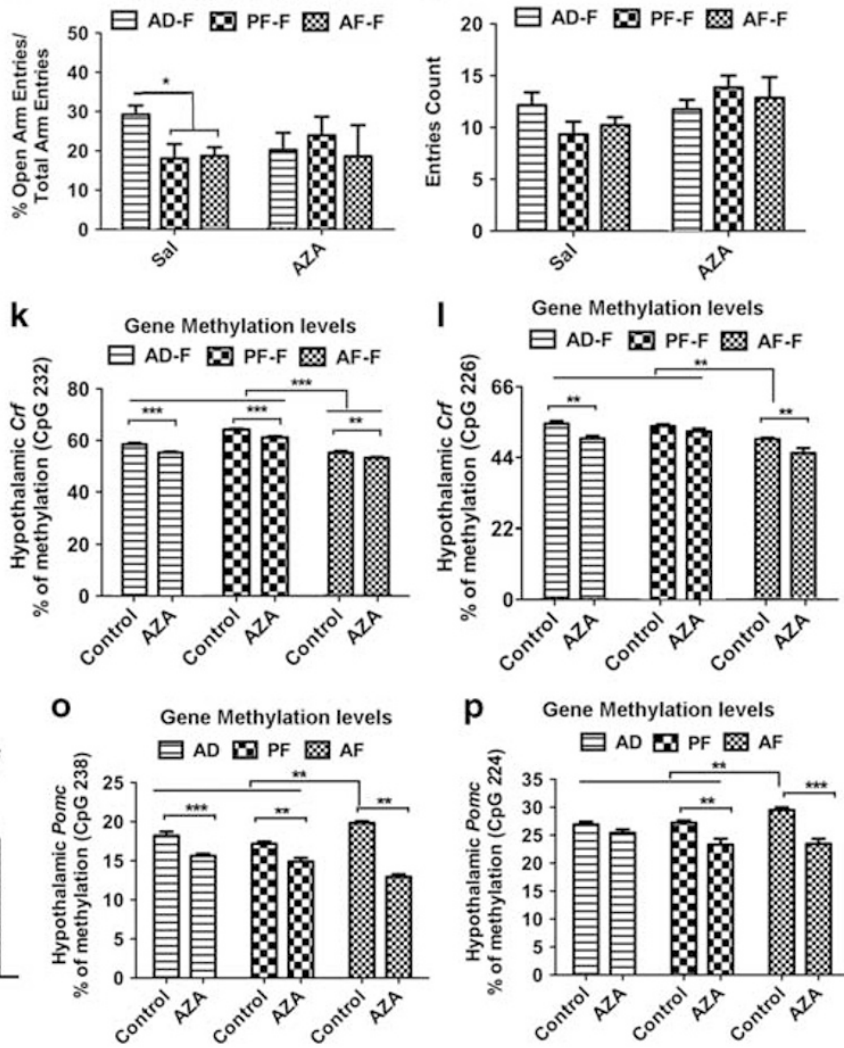

p Gene Methylation levels
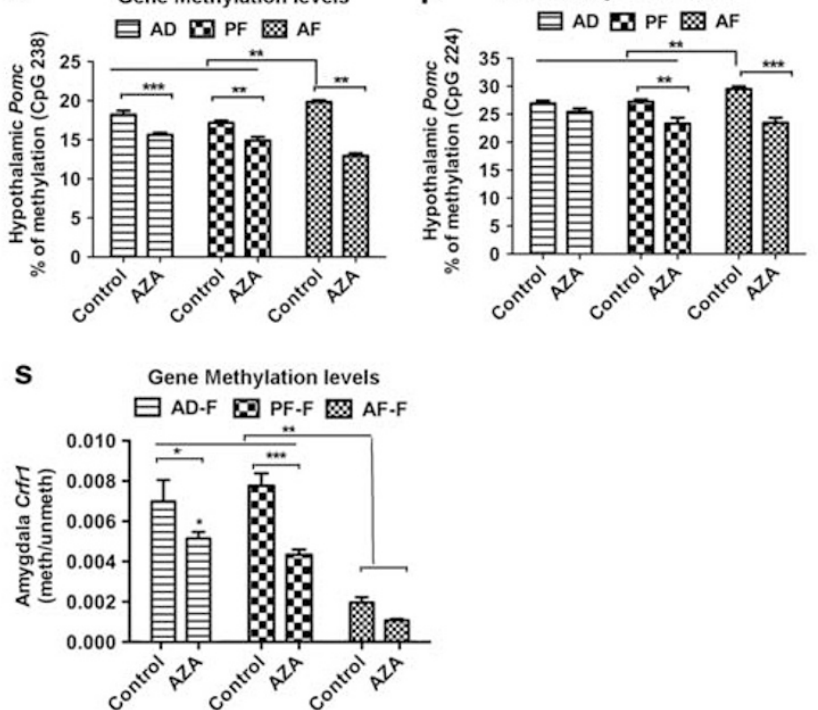

Figure 5 Suppression of DNA methylation prevents preconception alcohol effect on stress response, anxiety behaviors and stress regulatory gene expression. Treatment of AZA prevents the stress response (a, b, c, d), anxiety behaviors measured in OF (e) and in EPM (f, g, h), and the regulatory genes expressions (i, j, $\mathrm{m}, \mathrm{n}, \mathrm{q}, \mathrm{r})$, and methylation $(\mathrm{k}, \mathrm{l}, \mathrm{o}, \mathrm{p}, \mathrm{s})$ in AF-M or AF-F rats. Data are mean \pm SEM $(n=8$, except $n=18$ in all groups shown in $\mathrm{f}-\mathrm{h})$ and were analyzed by two-way ANOVA (alcohol $\times$ drug treatment) followed by Bonferroni post-test comparisons. $* p<0.05$, $* *$ * $p<0.01$, **** $p<0.001$, compared with saline-treated but differentially fed offspring for the gene measurements. * $p<0.05$, compared with saline-treated AFM for the gene measurements.

(Gangisetty et al, 2014; Lopez-Serra et al, 2008). In our experiments, Aza elicited its effect on stress regulatory genes by reducing their methylation and increasing expression. Hence, Aza effects on stress regulatory genes may be mediated by reducing the affinity of MeCP2. MeCP2 is also known to recruit histone modifying enzymes such as HDACs
(Jones et al, 1998; Wade et al, 1999) and HMTs (Fujita et al, 2003; Fuks et al, 2003). Hence, these epigenetic modifiers may also have roles in regulation of these HPA axis genes. It has been reported that early stress induced Crf expression was associated with increased $\mathrm{H} 3$ acetylation and reduced level of cytosine methylation at its promoter in hippocampus 
(Wang et al, 2014). Rats exposed to chronic mild stress associated with increased expression of Crfrl showed reduced levels of $\mathrm{H} 3 \mathrm{~K} 9$ trimethylation at the promoter in hypothalamus (Wang et al, 2014). Hence, further investigation is needed to evaluate whether, in addition to DNA methylation, other histone code mofications are involved in mediation of PCAE effects on the HPA axis. Also, in this study AZA treatment was given during postnatal period. Therefore, it is difficult to determine whether the effects of AZA are due to reversal of heritable epigenetic marks or simply inhibition of DNA methylation during postnatal development.

In summary, we have shown that maternal alcohol consumption three weeks prior to conception confers stress axis functional abnormalities in rat offspring. Alterations in stress axis functions were observed on multiple levels of analysis. It should be emphasized that rats were fed with alcohol three weeks, equivalent to five rat reproductive cycles, prior to conception. Thus, the effects we observed in the offspring were not due to a direct effect of alcohol on the offspring rather the effect was possibly inherited from the mother following alcohol abuse and passed on to the offspring. The results presented here suggest the intriguing, and potentially alarming, possibility that exposure to alcohol produces transmissible long-lasting epigenetic changes that result in profound alterations to the physiology of the offspring. In this regard, it is interesting to note that increased anxiety in humans is often correlated with alcohol consumption and that alcohol use/abuse does run in families (Kendler et al, 2015; Sjoerds et al, 2013; Morean et al, 2009). These human data tie the data of our present study in animal model in a translational way.

\section{FUNDING AND DISCLOSURE}

This work is partly supported by NIH grants R37AA08757, R01AA011591 to DKS, and F32AA023434 grant to LGC. The authors declare no conflict of interest.

\section{ACKNOWLEDGMENTS}

We thank Andy Mead for technical assistance in conducting gene expression and DNA methylation studies.

\section{REFERENCES}

Blizard DA, Lippman HR, Chen JJ (1975). Sex differences in openfield behavior in the rat: the inductive and activational role of gonadal hormones. Physiol Behav 14: 601-608.

Boschen KE, Ruggiero MJ, Klintsova AY (2016). Neonatal binge alcohol exposure increases microglial activation in the developing rat hippocampus. Neuroscience 324: 355-366.

Cameron N, Cameron NM, Champagne FA, Parent C, Fish EW, Ozaki-Kuroda $\mathrm{K}$ et al (2005). The programming of individual differences in defensive responses and reproductive strategies in the rat through variations in maternal care. Neurosci Biobehav Rev 29: 843-865.

Chen J, Evans AN, Liu Y, Honda M, Saavedra JM, Aguilera G (2012). Maternal deprivation in rats is associated with corticotrophin-releasing hormone $(\mathrm{CRH})$ promoter hypomethylation and enhances $\mathrm{CRH}$ transcriptional responses to stress in adulthood. J Neuroendocrinol 24: 1055-1064.
Dann CT, Alvarado AL, Hammer RE, Garbers DL (2006). Heritable and stable gene knockdown in rats. Proc Natl Acad Sci USA 103: $11246-11251$.

Dover GJ (2009). The Barker hypothesis: how pediatricans will diagnose and prevent common adult-onset diseases. Trans Am Clin Climatol Assoc 120: 199-207.

Ehrlich S, Weiss D, Burghardt R, Infante-Duarte C, Brockhaus S, Muschler MA et al (2010). Promoter specific DNA methylation and gene expression of POMC in acutely underweight and recovered patients with anorexia nervosa. J Psychiatr Res 44: 827-833.

Finegersh A, Rompala GR, Martin DI, Homanics GE (2015). Drinking beyond a lifetime: New and emerging insights into paternal alcohol exposure on subsequent generations. Alcohol 49: 461-470.

Fujita N, Watanabe S, Ichimura T, Tsuruzoe S, Shinkai Y, Tachibana $\mathrm{M}$ et al (2003). Methyl-CpG binding domain 1 (MBD1) interacts with the Suv39h1-HP1 heterochromatic complex for DNA methylation-based transcriptional repression. J Biol Chem 278: 24132-24138.

Fuks F, Hurd PJ, Wolf D, Nan X, Bird AP (2003). The methylCpGbinding protein MeCP2 links DNA methylation to histone methylation. J Biol Chem 278: 4035-4040.

Gangisetty O, Bekdash R, Maglakelidze G, Sarkar DK (2014). Fetal alcohol exposure alters proopiomelanocortin gene expression and hypothalamic-pituitary-adrenal axis function via increasing MeCP2 expression in the hypothalamus. PLOS ONE 9: e113228.

Gilpin NW, Herman MA, Roberto M (2015). The central amygdala as an integrative hub for anxiety and alcohol use disorders. Biol Psychiatry 77: 859-869.

Glavas MM, Ellis L, Yu WK, Weinberg J (2007). Effects of prenatal ethanol exposure on basal limbic-hypothalamic-pituitary-adrenal regulation: role of corticosterone. Alcohol Clin Exp Res 31: 1598-1610.

Govorko D, Bekdash RA, Zhang C, Sarkar DK (2012). Male germline transmits fetal alcohol adverse effect on hypothalamic proopiomelanocortin gene across generations. Biol Psychiatry 72: 378-388.

Hellemans KG, Verma P, Yoon E, Yu WK, Young AH, Weinberg J (2010). Prenatal alcohol exposure and chronic mild stress differentially alter depressive- and anxiety-like behaviors in male and female offspring. Alcohol Clin Exp Res 34: 633-645.

Johnston AL, File SE (1991). Sex differences in animal tests of anxiety. Physiol Behav 49: 245-250.

Jones PL, Veenstra GJ, Wade PA, Vermaak D, Kass SU, Landsberger $\mathrm{N}$ et al (1998). Methylated DNA and MeCP2 recruit histone deacetylase to repress transcription. Nat Genet 19: 187-191.

Kalil B, Leite CM, Carvalho-Lima M, Anselmo-Franci JA (2013). Role of sex steroids in progesterone and corticosterone response to acute restraint stress in rats: sex differences. Stress 16: 452-460.

Kendler KS, Edwards A, Myers J, Cho SB, Adkins A, Dick D (2015). The predictive power of family history measures of alcohol and drug problems and internalizing disorders in a college population. Am J Med Genet B Neuropsychiatr Genet 168B: $337-346$.

Lasebikan VO, Gureje O (2015). Lifetime and 7-day alcohol consumption in the elderly, prevalence and correlates: Reports from the Ibadan Study of Aging. Afr J Med Med Sci 44: 33-41.

Leshem M, Schulkin J (2012). Transgenerational effects of infantile adversity and enrichment in male and female rats. Dev Psychobiol 54: 169-186.

Li H, Zhang L, Fang Z, Lin L, Wu C, Huang Q (2010). Behavioral and neurobiological studies on the male progeny of maternal rats exposed to chronic unpredictable stress before pregnancy. Neurosci Lett 469: 278-282. 
Logan RW, Wynne O, Maglakelidze G, Zhang C, O'Connell S, Boyadjieva NI et al (2015). beta-Endorphin neuronal transplantation into the hypothalamus alters anxiety-like behaviors in prenatal alcohol-exposed rats and alcohol-non-preferring and alcohol-preferring rats. Alcohol Clin Exp Res 39: 146-157.

Lopez-Serra L, Ballestar E, Ropero S, Setien F, Billard LM, Fraga MF et al (2008). Unmasking of epigenetically silenced candidate tumor suppressor genes by removal of methyl-CpG-binding domain proteins. Oncogene 27: 3556-3566.

Malan-Müller S, Seedat S, Hemmings SM (2014). Understanding posttraumatic stress disorder: insights from the methylome. Genes Brain Behav 13: 52-68.

McEwen B, Gray JD, Nasca C (2015). Redefining neuroendocrinology: stress, sex and cognitive and emotional regulation. $J$ Endocrinol 226: T67-T83.

McGowan PO, Meaney MJ, Szyf M (2008). Diet and the epigenetic (re)programming of phenotypic differences in behavior. Brain Res 1237: 12-24.

Morean ME, Corbin WR, Sinha R, O'Malley SS (2009). Parental history of anxiety and alcohol-use disorders and alcohol expectancies as predictors of alcohol-related problems. J Stud Alcohol Drugs 70: 227-236.

National Instititute on Alcohol Abuse and Alcoholism (NIAAA) (2016). Alcohol and your health. Available at http://www.niaaa. nih.gov/alcohol-health/overview-alcohol-consumption/moderatebinge-drinking.

Nykjaer C, Alwan NA, Greenwood DC, Simpson NA, Hay AW, White KL et al (2014). Maternal alcohol intake prior to and during pregnancy and risk of adverse birth outcomes: evidence from a British cohort. J Epidemiol Community Health 68: 542-549.

Osborn JA, Yu C, Gabriel K, Weinberg J (1998). Fetal ethanol effects on benzodiazepine sensitivity measured by behavior on the elevated plus-maze. Pharmacol Biochem Behav 60: 625-633.

Paxinos G, Watson C (1982). The Rat Brain in Stereotaxic Coordinates. New York Academic Press Inc: New York, NY, USA.
Roeleveld N, Vingerhoets E, Zielhuis GA, Gabreëls F (1992). Mental retardation associated with parental smoking and alcohol consumption before, during, and after pregnancy. Prev Med 21: $110-119$.

Sarkar DK, Zhang C (2013). Beta-endorphin neuron regulates stress response and innate immunity to prevent breast cancer growth and progression. Vitam Horm 93: 263-276.

Sjoerds Z, van Tol MJ, van den Brink W, van der Wee NJ, Aleman A, Beekman AT et al (2013). Family history of alcohol dependence modulates functional neurophysiology in mood/ anxiety disorders. Psychol Med 43: 1487-1497.

Sotnikov SV, Markt PO, Malik V, Chekmareva NY, Naik RR, Sah A et al (2014). Bidirectional rescue of extreme genetic predispositions to anxiety: impact of $\mathrm{CRH}$ receptor 1 as epigenetic plasticity gene in the amygdala. Transl Psychiatry 11: e359.

Substance Abuse and Mental Health Services Administration (SAMHSA) (2014). National Survey on Drug Use and Health (NSDUH). Available at http://www.samhsa.gov/data/sites/default/ files/NSDUH-DetTabs2014/NSDUH-DetTabs2014.htm\#tab5-8b.

Tu W, Cook A, Scholl JL, Mears M, Watt MJ, Renner KJ et al (2014). Serotonin in the ventral hippocampus modulates anxiety-like behavior during amphetamine withdrawal. Neuroscience 281C: 35-43.

Vaissière T, Sawan C, Herceg Z (2008). Epigenetic interplay between histone modifications and DNA methylation in gene silencing. Mutat Res 659: 40-48.

Wade PA, Gegonne A, Jones PL, Ballestar E, Aubry F et al (1999). Mi-2 complex couples DNA methylation to chromatin remodelling and histone deacetylation. Nat Genet 23: 62-66.

Wang A, Nie W, Li H, Hou Y, Yu Z, Fan Q et al (2014). Epigenetic upregulation of corticotrophin-releasing hormone mediates postnatal maternal separation-induced memory deficiency. PLOS ONE 9: e94394.

Wang X, Meng F, Liu Z, Fan J, Hao K, Chen X et al (2013). Gestational hypoxia induces sex differential methylation of Crhr1 linked to anxiety like behavior. Mol Neurobiol 48: 544-555.

Supplementary Information accompanies the paper on the Neuropsychopharmacology website (http://www.nature.com/npp) 